\title{
Tiam1 is Critical for Glutamatergic Synapse Structure and Function in the Hippocampus
}

\author{
Sadhna Rao, ${ }^{1}$ Yuni Kay, ${ }^{1}$ and Bruce E. Herring ${ }^{1,2}$ \\ ${ }^{1}$ Department of Biological Sciences, and ${ }^{2}$ The Bridge Institute, University of Southern California, Los Angeles, California 90089
}

\begin{abstract}
Mounting evidence suggests numerous glutamatergic synapse subtypes exist in the brain, and that these subtypes are likely defined by unique molecular regulatory mechanisms. Recent work has identified substantial divergence of molecular composition between commonly studied Schaffer collateral synapses and perforant path-dentate gyrus (DG) synapses of the hippocampus. However, little is known about the molecular mechanisms that may confer unique properties to perforant path-DG synapses. Here we investigate whether the RhoGEF (Rho guanine-nucleotide exchange factor) protein Tiam1 plays a unique role in the regulation of glutamatergic synapses in dentate granule neurons using a combination of molecular, electrophysiological, and imaging approaches in rat entorhino-hippocampal slices of both sexes. We find that inhibition of Tiam 1 function in dentate granule neurons reduces synaptic AMPA receptor function and causes dendritic spines to adopt an elongated filopodia-like morphology. We also find that Tiaml's support of perforant path-DG synapse function is dependent on its GEF domain and identify a potential role for the auto-inhibitory PH domain of Tiam1 in regulating Tiaml function at these synapses. In marked contrast, reduced Tiam1 expression in CA1 pyramidal neurons produced no effect on glutamatergic synapse development. Together, these data identify a critical role for Tiam1 in the hippocampus and reveal a unique Tiam1-mediated molecular program of glutamatergic synapse regulation in dentate granule neurons.
\end{abstract}

Key words: dentate gyrus; hippocampus; maturation; RhoGEF; synapse; Tiam1

Significance Statement

Several lines of evidence independently point to the molecular diversity of glutamatergic synapses in the brain. Rho guaninenucleotide exchange factor (RhoGEF) proteins as powerful modulators of glutamatergic synapse function have also become increasingly appreciated in recent years. Here we investigate the synaptic regulatory role of the RhoGEF protein Tiaml, whose expression appears to be remarkably enriched in granule neurons of the dentate gyrus. We find that Tiaml plays a critical role in the development of glutamatergic perforant path-dentate gyrus synapses, but not in commonly studied in Schaffer collateralCA1 synapses. Together, these data reveal a unique RhoGEF-mediated molecular program of glutamatergic synapse regulation in dentate granule neurons.

\section{Introduction}

Glutamatergic synapse maturation and function are governed by a diverse assortment of synaptic molecules. Of these, Rho guanine-nucleotide exchange factor (RhoGEF) proteins have been increasingly implicated in supporting glutamatergic synapse

\footnotetext{
Received July 2, 2019; revised Oct. 2, 2019; accepted Oct. 3, 2019.

Author contributions: S.R. and B.E.H. designed research; S.R. and Y.K. performed research; S.R. analyzed data; S.R. and B.E.H. wrote the paper.

This work was supported by McKnight, Simons (503736), and Brain and Behavior Research Foundation awards to B.E.H. and the U.S. National Institute of Mental Health (Grant MH-103398 to B.E.H). All primary data are archived in the Department of Biological Sciences, University of Southern California. We thank the members of the Herring laboratory for helpful feedback and comments during the study and for technical assistance with slice preparation and culture. We also thank Dr. Judith Hirsch for assistance in preparing this manuscript.

The authors declare no competing financial interests.

Correspondence should be addressed to Bruce E. Herring at bherring@usc.edu.

https://doi.org/10.1523/JNEUROSCI.1566-19.2019

Copyright $\odot 2019$ the authors
}

structure and function through their ability to catalyze actin polymerization (Kang and Schuman, 1995; Penzes et al., 2003, 2008; Fu et al., 2007; Xie et al., 2007; Margolis et al., 2010; Miller et al., 2013; Ryan et al., 2015; Herring and Nicoll, 2016; Hamilton et al., 2017; Martin-Vilchez et al., 2017). However, efforts to characterize the role of RhoGEFs and other synaptic proteins have been largely restricted to synapses between neurons of unknown identity in dissociated neuronal preparations or to Schaffer collateralCA1 synapses, which are used as models for all glutamatergic synapses. This reductionist approach to studying synaptic proteins overlooks a growing body of evidence that points to the existence of heterogeneous populations of glutamatergic synapses in the brain (Zhao et al., 2001; Datson et al., 2004, 2009; Lein et al., 2004; Coultrap et al., 2005).

Recent whole-brain synaptome cartography has identified substantial divergence in molecular composition between commonly studied CA3-CA1 Schaffer collateral synapses and ento- 
rhinal cortex-dentate gyrus (DG) perforant path synapses of the hippocampus (Zhu et al., 2018). Such data suggest that pathwayspecific molecular programs regulating glutamatergic synapse maturation and function are likely to exist. In fact, little is known of the unique molecular mechanisms that may operate within perforant path-DG synapses. This pathway serves as the primary gateway of information flow from the cortex to the hippocampus and delivers glutamatergic input to granule neurons of the dentate gyrus (DG granule neurons).

Interestingly, the RhoGEF protein Tiam1 has substantially higher transcript expression in DG granule neurons relative to CA3 and CA1 pyramidal neurons (Ehler et al., 1997; Lein et al., 2004), predicting possible differential influence between hippocampal subregions. Although previous reports have implicated endogenous Tiam1 in synapse regulation (Chen and Macara, 2005; Tolias et al., 2005, 2007; Zhang and Macara, 2006; Um et al., 2014), the synaptic role of Tiam1 in DG granule neurons has not been explored.

In this study, we examine whether Tiam1 plays a pathwayspecific role in the regulation of synaptic transmission in the hippocampus. We use a combination of molecular, imaging, and electrophysiological techniques to compare the role of Tiam1 in perforant path-DG and Schaffer collateral-CA1 hippocampal synapses. We find that the inhibition of Tiam1 function in DG granule neurons results in a significant and selective reduction in synaptic AMPA receptor function that is accompanied by dendritic spines adopting an elongated and filopodial appearance. In marked contrast, inhibition of Tiam 1 in CA1 pyramidal neurons produced no detectable effect on glutamatergic synapse development.

Previous work has suggested that Tiam1 regulates actin polymerization in dendritic spines via activation of the small GTPase Rac1 through its GEF domain (Penzes and Rafalovich, 2012; Duman et al., 2013). However, a recent study delineates a GEF-independent mechanism by which Tiam1 regulates neurons (Tang et al., 2019). Here, we find that the GEF/DH (Dblhomology) domain of Tiam 1 is essential for its influence on perforant path-DG synapse development. We also find that the Pleckstrin homology coiled-coil extra $\left(\mathrm{PH}_{\mathrm{n}}-\mathrm{CC}-\mathrm{Ex}\right)$ domain of Tiam1 -an auto-inhibitory region that restricts the GEF activity of Tiam1- negatively regulates Tiam1 function at DG granule synapses. Together, our data identify a pathway-specific and GEF-dependent role for Tiam1 in the hippocampus and reveal a unique RhoGEF-mediated molecular program of glutamatergic synapse regulation in DG granule neurons.

\section{Materials and Methods}

Experimental constructs. A previously characterized Tiam1 shRNA target sequence against rat Tiam1 was used (5'-GAGGGAGAAGGAAGTG GTCT-3'; Tolias et al., 2005). The Tiam1 shRNA was subcloned behind the $\mathrm{H} 1$ promoter region of a GFP-expressing pFHUGW expression vector. The human Tiam 1 cDNA sequence was acquired from a construct containing human Tiam1 cDNA (accession \#BC117196, Open Biosystems).

The shRNA-resistant Tiam1 was generated by introducing five silent point mutations within the RNAi target sequence (AAGAGAAAAA GAGGTGGTCT). All cloning was performed using overlap-extension PCR followed by Clontech In-Fusion Cloning (Takara Bio). Both human Tiam 1 cDNA and shRNA-resistant Tiam1 cDNA were cloned into a pCAGGS-IRES-mCherry expression vector and coexpressed with a pFUGW vector containing GFP for easy visualization of transfected neurons. The Tiam $1 \Delta \mathrm{DH}$ mutant was generated by deleting the $\sim 200$ residue DH domain (Lys1040-Glu1233) using overlap-extension PCR followed by Clontech In-Fusion Cloning (TaKaRa) into a pCAGGS-
IRES-mCherry expression vector and coexpressed with a pFUGW vector containing GFP. The Tiam $1 \Delta \mathrm{PH}_{\mathrm{n}}$-CC-Ex mutant was generated by deleting the $\sim 300$ residue $\mathrm{PH}_{\mathrm{n}}$-CC-Ex domain (Ala428-Thr702) using the same method as described above. The GFP construct also served as a control vector for spine-imaging experiments.

Immunohistochemistry. Postnatal day 7 (P7) rat hippocampi were dissected and fixed in 4\% paraformaldehyde (PFA) in $1 \times$ PBS for $30 \mathrm{~min}$, followed by overnight suspension in $60 \%$ sucrose in $1 \times$ PBS. The tissue was then frozen in cryoprotectant freezing media (catalog \#72592, Electron Microscopy Services) and sectioned into $15 \mu \mathrm{m}$ slices at $-20^{\circ} \mathrm{C}$. Tissue sections were mounted on microscope slides and probed with antibodies against Tiam1 (1:100; sheep anti-Tiam1; catalog \#AF5038, R\&D Systems) and Prox1 (1:2000; anti-Prox1; catalog \#ab5475, Millipore). Fluorophore-coupled secondary antibodies were used for detection, and slides were imaged using a Zeiss model 510 Confocal Microscope equipped with an EC Plan Neofluoar $40 \times / 1.3$ oil-immersion differential interference contrast (DIC) objective. The $z$-stacks were captured at $0.5 \mu \mathrm{m}$ intervals and were processed to create maximum intensity projections.

Electrophysiology. Organotypic entorhino-hippocampal slice cultures were prepared from P6 to P8 Sprague Dawley rats of both sexes as previously described (Stoppini et al., 1991; Prang et al., 2001; Bonnici and Kapfhammer, 2009). Tissue was isolated and a MX-TS tissue slicer (Siskiyou) was used to make $400 \mu \mathrm{m}$ transverse sections. Tissue slices were placed on squares of Biopore Membrane Filter Roll (Millipore) and placed on Millicell Cell Culture inserts (Millipore) in $35 \mathrm{~mm}$ dishes. The slices were fed on alternate days with $1 \mathrm{ml}$ of culture media (Invitrogen MEM + HEPES; catalog \#12360-038, Thermo Fisher Scientific; horse serum (25\%); HBSS (25\%); and L-glutamine $1 \mathrm{~mm}$ ).

Sparse biolistic transfections were performed on day in vitro 1 (DIV1) as previously described (Stoppini et al., 1991; Schnell et al., 2002; Lu et al., 2009). Recordings were made on DIV7 or DIV9 in slice cultures on an upright Olympus BX50WI Microscope and perfused at $2.5 \mathrm{ml} \mathrm{min}^{-1}$ with artificial CSF (aCSF) containing $119 \mathrm{~mm} \mathrm{NaCl}, 2.5 \mathrm{~mm} \mathrm{KCl}, 1 \mathrm{~mm}$ $\mathrm{NaH}_{2} \mathrm{PO}_{4}, 26.2 \mathrm{~mm} \mathrm{NaHCO}, 11 \mathrm{~mm}$ glucose, $4 \mathrm{~mm} \mathrm{CaCl}_{2}$, and $4 \mathrm{~mm}$ $\mathrm{MgSO}_{4}$ adjusted to osmolality of 305-315 mOsm, supplemented with 5 $\mu \mathrm{M} 2$-chloroadenosine to dampen epileptiform activity and $0.1 \mathrm{~mm}$ picrotoxin to block $\mathrm{GABA}_{\mathrm{A}}$ receptors. Borosilicate recording electrodes were filled with an internal solution containing $135 \mathrm{mM} \mathrm{CsMeSO}_{4}, 8 \mathrm{~mm}$ $\mathrm{NaCl}, 10$ mM HEPES, 0.3 mм EGTA, 5 mm QX-314, 4 mm Mg-ATP, and $0.3 \mathrm{~mm} \mathrm{Na}$-GTP adjusted to $\mathrm{pH} 7.3-7.4$ and osmolarity of 290-295 mOsm. The aCSF was bubbled with $95 \%(\mathrm{v} / \mathrm{v}) \mathrm{O}_{2}$ and $5 \%(\mathrm{v} / \mathrm{v}) \mathrm{CO}_{2}$ to maintain $\mathrm{pH}$.

DG granule neurons and CA1 pyramidal neurons were identified using differential interference phase contrast microscopy, while GFPexpressing transfected cells were identified using epifluorescence microscopy. Postsynaptic currents were elicited by stimulation of either stratum radiatum or perforant pathway afferents with a monopolar glass electrode. Membrane voltage was held at $-70 \mathrm{mV}$ to measure AMPARevoked EPSCs (eEPSCs), and at $+40 \mathrm{mV}$ to measure NMDAR-eEPSCs, both of which were usually measured from the same paired recording. No more than one pair was recorded from a single entorhinohippocampal slice. Membrane holding current, pipette series resistance, and input resistance were monitored throughout recording sessions. Data were gathered through a MultiClamp 700B amplifier (Molecular Devices), filtered at $2 \mathrm{kHz}$, and digitized at $10 \mathrm{kHz}$.

Coefficient of variation $(\mathrm{CV})$ analysis was performed on AMPAReEPSCs by calculating the mean (M) and standard deviation (SD) (Kokaia et al., 1998) of 20 consecutively recorded current amplitudes for both control and transfected cells within a pair from a dual whole-cell patch-clamp recording. From several such pairs, the $\mathrm{CV}$ was calculated as $\mathrm{SD} / \mathrm{M}$. It has been shown both theoretically and experimentally that $\mathrm{CV}^{-2}\left(\mathrm{M}^{2} / \mathrm{SD}^{2}\right)$ is invariant with changes in quantal size (i.e., the number of AMPA receptors at all synapses) and that $\mathrm{CV}^{-2}$ varies predictably with changes in quantal content (i.e., the number of functional synapses containing AMPA receptors) according to the following equation $\mathrm{CV}^{-2}$ $=n \times \operatorname{Pr} /(1-\operatorname{Pr})$ where $n$ is the number of vesicle release sites, and $\operatorname{Pr}$ is the probability of presynaptic release. To compare the eEPSC variance with changes in mean amplitude, the $\mathrm{CV}^{-2}$ values for transfected and control cells were plotted on the $y$-axis, against the ratios of means for 
transfected and control cells that were plotted on the $x$-axis. Values above the $45^{\circ}(y=x)$ line indicate increases in quantal content, while values approaching the horizontal line $(y=1)$ indicate a change in quantal size as ultimately responsible for the difference in AMPAR-eEPSC amplitude between the control and transfected cells.

Failure analysis was performed by analyzing AMPAR-eEPSCs from dual whole-cell patch-clamp recordings where stimulation levels elicited failures that could be easily distinguished from currents by eye. Events were assigned as failures if their absolute magnitudes were less than or equal to noise for each sweep. The number of failures for each cell was estimated as the number of events with absolute current amplitude greater than noise divided by the total number of events to yield the percentage failure rate.

Imaging and spine analysis. Cultured entorhino-hippocampal slices were transfected with pFHUGW-GFP shRNA constructs alone or pFHUGW-GFP shRNA and pCAGGS-mCherry cDNA constructs $\sim 18-$ $20 \mathrm{~h}$ after plating using biolistic transfection. The experimenter was blinded to genotype during subsequent processing and imaging. Slices were fixed in 4\% PFA, 4\% sucrose in PBS, and washed three times with PBS, then cleared with an abbreviated SeeDB-based protocol (Ke et al., 2013) and mounted on microscope slides. High-resolution confocal $z$-stacks of spine-containing DG granule neuron apical dendrites and CA1 pyramidal neuron apical dendrites were acquired on a Zeiss 510 using a Plan Apochromat $63 \times / 1.4$ oil-immersion DIC objective. The $z$-stacks were collected at maximum X-Y pixel dimensions $(2048 \times 2048$ pixels) at 12 bits with $X-Y$ spatial resolution of $70 \mathrm{~nm}$ and axial resolution of $500 \mathrm{~nm}$ with a $488 \mathrm{~nm}$ laser excitation wavelength. Automated analysis of dendritic segments and spines was performed using the commercially available software Filament Tracer (Imaris 9.1.2, Bitplane). For each cell, an $\sim 60 \mu \mathrm{m}$ dendritic segment was manually selected for analysis, and thresholds for dendritic surface and spine rendering were set (minimum spine diameter and maximum spine length were set to 0.2 and $10 \mu \mathrm{m}$, respectively). Data were exported into Microsoft Excel and graphed using R Studio (version 1.1.423).

Western blotting. Embryonic day 16.5 hippocampi were dissected, dissociated, and cultured in DMEM with $10 \%$ FBS. The neurons were plated onto six-well plates and treated with $20 \mu \mathrm{l}$ virus (pFUGW-GFP lentivirus or pFHUGW-H1-Tiam1shRNA-UbC-GFP lentivirus) at DIV0, and at DIV21 cell lysates were prepared. In experiments involving the expression of Tiam 1 and Tiam $1 \Delta \mathrm{DH}$ in HEK293T cells (ATCC), cells were transfected with $2 \mu \mathrm{g}$ of DNA (pFUGW-GFP alone, pFUGW-GFP and pCAGGS-Tiam1- $\triangle \mathrm{DH}$-IRES-mcherry, pFUGW-GFP, and pCAGGSTiam-IRES-mcherry), and cell lysates were prepared after $72 \mathrm{~h}$ of expression. For knock-down experiments in HEK cells, a Tiam1 shRNA/Tiam1 cDNA DNA ratio of 20:1 was used, and lysates were prepared $40 \mathrm{~h}$ later. All lysates were run on a 4-15\% Mini-PROTEAN TGX Precast Protein Gel (Bio-Rad) with $50 \mu \mathrm{g}$ of protein loaded per lane. Membranes were probed with antibodies specific for Tiam1 (1:100; sc-393315, Santa Cruz Biotechnology) and $\beta$-actin (1:1000; (13E5) Rabbit mAb; catalog \#4970, Cell Signaling Technology). Horseradish peroxidase-coupled secondary antibodies were then used for detection. Membranes were scanned using the Bio-Rad Chemidoc Imaging System.

Experimental design and statistical analysis. All electrophysiological data are expressed as the mean \pm SEM. For all experiments, at least three male and female rat pups were used. All imaging analysis was performed blind to genotype. Statistical significance was determined using the Wilcoxon signed-rank test for paired dual whole-cell patch-clamp data, Wilcoxon rank-sum test for imaging data, and Student's $t$ test for pairedpulse facilitation (PPF) data. For RNA sequencing (RNA-seq) data in hippocampal subregions, post hoc analysis was performed on data downloaded from the Hipposeq RNA-seq Atlas (Cembrowski et al., 2016) and imported into R Studio (version 1.1.423). For $\mathrm{CV}^{-2}$ analysis, linear regression analysis was performed using the least-squares method with GraphPad Prism. All $p$ values $<0.05$ were considered significant and denoted with a single asterisk, $p$ values $<0.01$ were denoted with a double asterisk, and $p$ values $<0.001$ were denoted with a triple asterisk. All error bars represent SE measurement. Sample sizes in the present study are similar to those reported in the literature (Herring and Nicoll, 2016; Incontro et al., 2018).

\section{Results}

\section{Tiam1 is required for AMPAR-mediated neurotransmission} in DG granule neurons

In situ hybridization and RNA-seq data show a significantly higher level of Tiam $1 \mathrm{mRNA}$ expression in the DG relative to CA1 and CA3 subregions of the hippocampus [Lein et al., 2006; Cembrowski et al., 2016; DG vs CA1, $p=0.0014$; DG vs CA3, $p=$ 0.0013; one-way ANOVA and post hoc Tukey HSD; Fig. $1 A, B$ (modified from Allen Brain Atlas)]. As expected, we observe robust Tiam1 protein expression in Prox1-positive DG granule neurons in hippocampal slices (Fig. 1C). To examine whether Tiam 1 depletion affects glutamatergic neurotransmission in the DG, we generated a Tiam1 shRNA construct based on a previously validated Tiam 1 shRNA target sequence (Tolias et al., 2005). We find that this Tiam 1 shRNA construct produces a substantial reduction of recombinant Tiam1 expression in HEK293 cells and endogenous Tiam1 in hippocampal neurons (Fig. 1D). Then we biolistically transfected DG granule neurons in rat organotypic entorhino-hippocampal slice cultures (Schnell et al., 2002; Elias et al., 2008) with our Tiam1 shRNA. Six days after transfection, a dual whole-cell patch-clamp approach was used to simultaneously measure AMPA and NMDA receptormediated eEPSCs (AMPAR-EPSCs and NMDAR-eEPSCs) in transfected and neighboring untransfected DG granule neurons in response to perforant pathway stimulation (Fig. 1E). This simultaneous pairwise measurement of currents from both transfected and untransfected control neurons allows an internally controlled test of the genetic manipulation. Using this approach, we found that DG granule neurons expressing Tiam1 shRNA exhibited an $\sim 50 \%$ reduction $(n=12$ pairs, $p=0.00049$, Wilcoxon signed-rank test) in average AMPAR-eEPSC amplitude compared with paired control neurons (Fig. $1 F$ ). A significant effect on NMDAR-eEPSC current amplitudes was not observed ( $n=12$ pairs, $p=0.09$, Wilcoxon signed-rank test; Fig. $1 G$ ). Together, these data establish Tiam 1 as an important regulator of synaptic AMPAR-mediated glutamatergic neurotransmission at perforant path-DG synapses.

To determine whether of Tiam1 in DG granule neurons alters presynaptic neurotransmitter release, we examined PPF in neurons transfected with Tiam 1 shRNA and neighboring control neurons. We observed no changes in paired-pulse facilitation following the knockdown of Tiam1 ( $p=0.216, n=7$, Student's $t$ test; Fig. $1 H$ ). Thus, the synaptic effects we observe in DG granule neurons are due to alteration of the postsynaptic side of the synapse.

Following Tiam1 knockdown, we observe a reduction in synaptic AMPAR function but not NMDAR function. This selective reduction in AMPAR function may be caused by a reduction in AMPARs across all functional glutamatergic synapses or arise from a subset of functional synapses losing all their AMPARs and thus becoming "silent synapses." To determine which of these possibilities had occurred, we first performed coefficient of variation analysis on AMPAR-eEPSC current amplitudes. Coefficient of variation analysis can be used to determine the quantal parameters of glutamatergic transmission in control and transfected neurons. By comparing the normalized variance in AMPAR-eEPSC amplitudes from two neurons receiving the same stimulus, it is possible to estimate relative quantal size and quantal content (del Castillo and Katz, 1954; Bekkers and Stevens, 1990; Malinow and Tsien, 1990; Gray et al., 2011; Levy et al., 2015). Changes in quantal size precisely change both the mean eEPSC and the variance such that the normalized ratio of mean ${ }^{2} /$ 
A

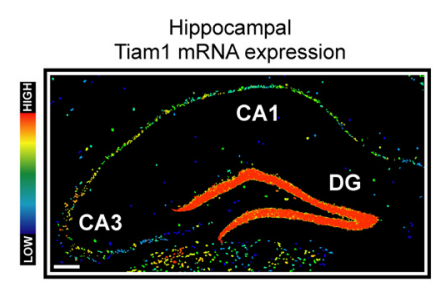

F
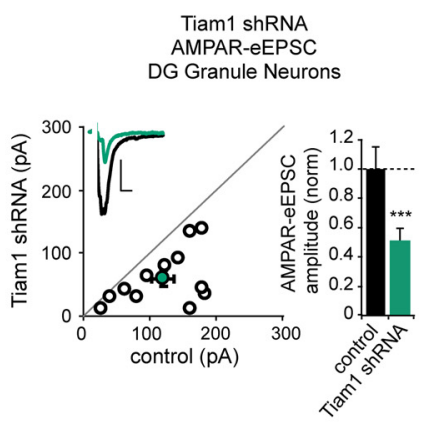

G
B

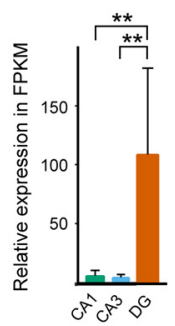

C

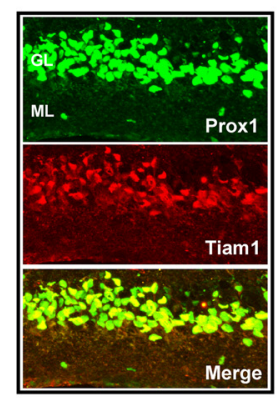

D

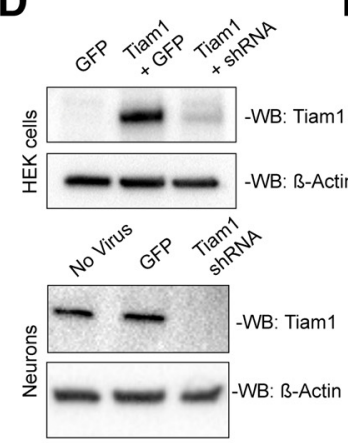

E

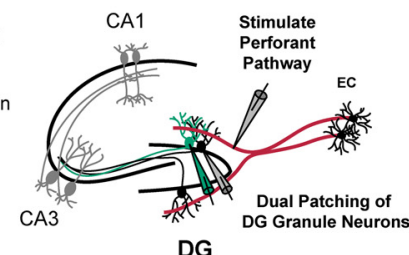

H

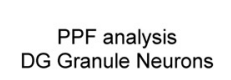

I

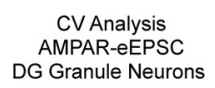

J

Failure Analysis AMPAR-eEPSCs DG Granule Neurons
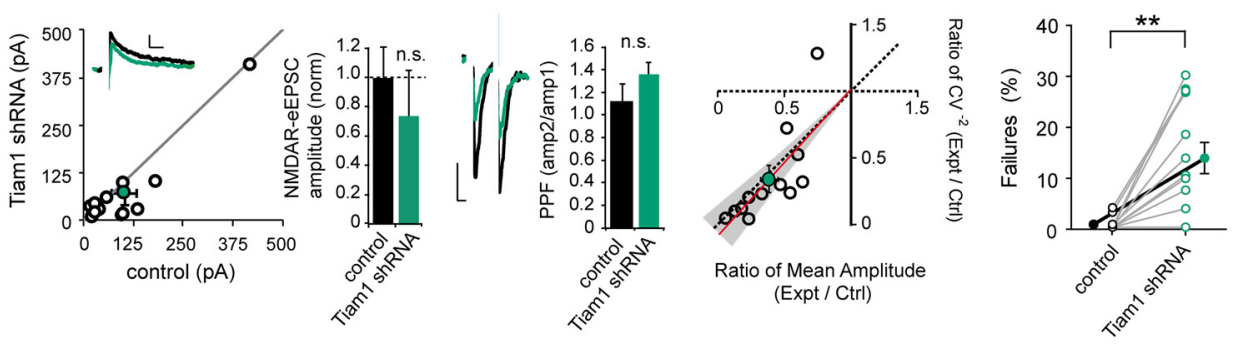

Figure 1. Tiam 1 knockdown reduces AMPAR-mediated neurotransmission in DG granule neurons. A, Hippocampal Tiam1 mRNA expression data from the Allen Mouse Brain Atlas. Scale bar, 200 $\mu \mathrm{m} . \boldsymbol{B}$, Tiam1 RNA sequencing data in CA1 and CA3 pyramidal neurons and DG granule neurons from the Hipposeq RNA-seq database. ${ }^{* *} p<0.01$, one-way ANOVA with post hoc Tukey HSD. C, Immunolabeling of Prox1 and Tiam1 in DG granule neurons in hippocampal slices. GL, granule layer; ML, molecular layer. $D$, Western blot showing shRNA-mediated reduction of Tiam1 expression in HEK293 cells (top) and hippocampal neurons (bottom). $\boldsymbol{E}$, Schematic representation of electrophysiological recording setup for DG granule neurons. $\boldsymbol{F}, \boldsymbol{G}$, Scatterplots show eEPSC amplitudes for pairs of untransfected and transfected cells (open circles) with corresponding mean \pm SEM (filled circles). Insets, Representative current traces from control and transfected (green) neurons with stimulation artifacts removed. Calibration: 20 pA for both AMPAR-eEPSC and NMDAR-eEPSC, $20 \mathrm{~ms}$ for AMPAR-eEPSC, $50 \mathrm{~ms}$ for NMDAR-eEPSC. Barplots show the average AMPAR-eEPSC and NMDAR-eEPSC amplitudes ( \pm SEM) of DG granule neurons expressing Tiam1 shRNA normalized to their respective control cell average eEPSC amplitudes. Tiam1 shRNA expression decreases AMPAR-eEPSC amplitude in DG granule neurons ( $n=12$ pairs) but has no detectable effect on NMDAR-eEPSC amplitude $\left(n=12\right.$ pairs). ${ }^{* * *} p<0.001$; . n.s., Not significant, Wilcoxon signed-rank test. $\boldsymbol{H}$, Paired-pulse facilitation ratios (mean \pm SEM) for Tiam1 shRNA expressing DG granule neurons and paired control neurons with no detectable difference in facilitation ( $n=7$ pairs). $n$.s., Not significant, Student's $t$ test. Representative scaled current traces from control and transfected (green) neurons. Calibration: 20 pA, 20 ms. I, Coefficient of variation analysis of AMPAR-eEPSCs from pairs of control and Tiam1 shRNA expressing DG granule neurons. $\mathrm{CV}^{-2}$ values are plotted against corresponding ratios of mean amplitudes within each pair (open circles) with mean \pm SEM (filled circle). Red line represents "best-fit" linear regression, and gray-shaded area indicates $95 \%$ confidence interval for regression ( $n=12$ pairs).J, Failure analysis of AMPAR-eEPSCs from pairs of control and Tiam 1 shRNA-expressing DG granule neurons with DG granule neurons exhibiting higher failure rates than control neurons ( $n=12$ pairs). ${ }^{* *} p<0.01$, Wilcoxon signed-rank test.

variance, also known as coefficient of variation $\left(\mathrm{or} \mathrm{CV}^{-2}\right)$, remains constant. Changes in quantal size cause the marker of the mean to fall on the horizontal line seen in Figure $1 I$ and, in the context of this preparation, indicate a change in the number of glutamate receptors at all synapses. In contrast, changes in quantal content will produce proportional changes of equal magnitude in $\mathrm{CV}^{-2}$ and mean eEPSC amplitudes that cause the marker of the mean to fall on the diagonal line. Here, changes in quantal content indicate a change in the number of synapses expressing glutamatergic receptors. We observed proportional reductions in $\mathrm{CV}^{-2}$ and mean AMPAR-eEPSC amplitude following Tiam1 knockdown in DG granule neurons. This result identified a clear reduction in quantal content rather than quantal size as responsible for the reduction of AMPAR-eEPSC amplitude we observe (Fig. 1I). Such data suggest that knocking down Tiam1 results in a reduction in the number of synapses that contain AMPARs.

To more directly determine whether Tiam 1 knockdown reduces the number of glutamatergic synapses that contain AMPARs, we performed failure analysis of AMPAR-eEPSCs from Tiam1 knock-down neurons and neighboring control neurons. Changes in AMPAR-eEPSC failure rate are produced by alterations of the number of synapses containing AMPARs (Goold and Nicoll, 2010; Gray et al., 2011). Consistent with our
CV analysis, we find that knocking down Tiam1 results in a significant increase in the number of failures we observe relative to control cells $(p=0.0059, n=12$, Student's $t$ test; Fig. $1 J)$. Together, our findings strongly suggest that reducing Tiam1 expression in DG granule neurons decreases the number of glutamatergic synapses that express AMPARs.

\section{AMPAR- and NMDAR-mediated neurotransmission are normal in CA1 pyramidal neurons following Tiam1 knockdown}

In situ hybridization and RNA-seq data for Tiam1 shows high transcript expression levels in the DG, but considerably lower levels in other hippocampal subregions (Figs. $1 A, B, 2 A$ ). While we observed robust Tiam1 protein expression in DG granule neurons, Tiam1 expression in CA1 pyramidal neurons was below the detection threshold of our antibody (Fig. 2B). Consistent with the Tiam1 mRNA and protein expression data, we observed no detectable differences in AMPAR-eEPSC or NMDAR-eEPSC amplitudes in Tiam 1 shRNA expressing CA1 pyramidal neurons following Schaffer collateral stimulation (for AMPAR-eEPSC: $p=0.8, n=9$ pairs; for NMDAR-eEPSC: $p=0.5, n=8$ pairs, Wilcoxon signed-rank test; Fig. $2 C-E$ ). Together, these data sug- 
A

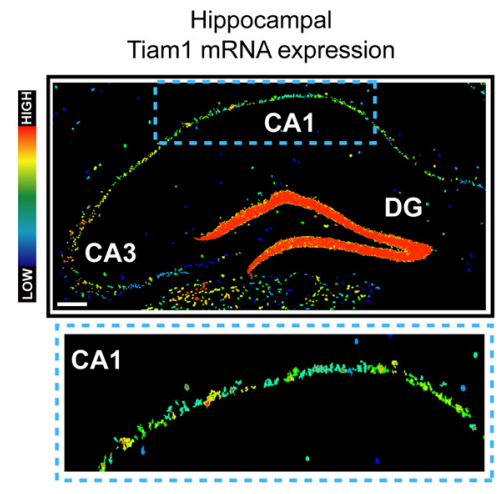

\section{C}

CA1 Pyramidal Neurons

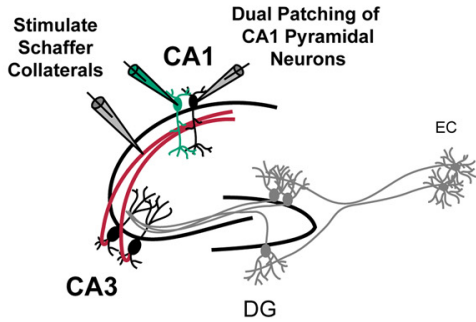

D

B

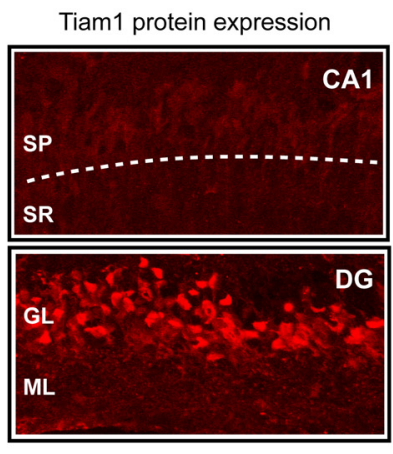

E

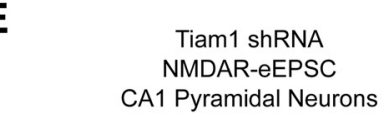

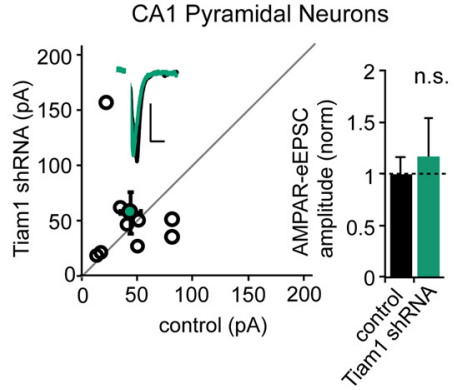

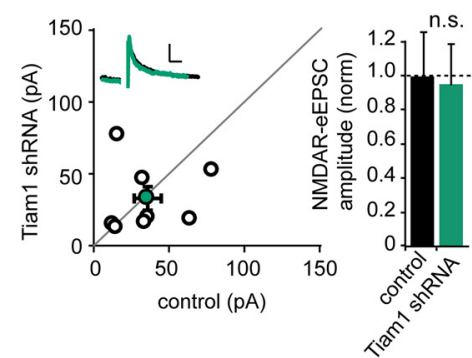

Figure 2. Tiam 1 knockdown does not affect AMPAR- or NMDAR-mediated neurotransmission in CA1 pyramidal neurons. $A$, Hippocampal Tiam $1 \mathrm{mRNA}$ expression data from the Allen Mouse Brain Atlas. Scale bar, $200 \mu \mathrm{m}$. Dashed blue box shows enlarged CA1 region. $\boldsymbol{B}$, Immunolabeling of Tiam1 in CA1 pyramidal neurons (top) and DG granule neurons (bottom) in hippocampal slices. SP, stratum pyramidale; SR, stratum radiatum; GL, granule layer; ML, molecular layer. C, Schematic representation of electrophysiological recording setup for CA1 pyramidal neurons. $\boldsymbol{D}, \boldsymbol{E}, \mathbf{S}$ catterplots show eEPSC amplitudes for pairs of untransfected and transfected cells (open circles) with corresponding mean \pm SEM (filled circles). Insets, Representative current traces from control and transfected (green) neurons with stimulation artifacts removed. Calibration: $20 \mathrm{pA}$ for both AMPAR-eEPSC and NMDAR-eEPSC, $20 \mathrm{~ms}$ for AMPAR-eEPSC, $50 \mathrm{~ms}$ for NMDAR-eEPSC. Barplots show the average AMPAR-eEPSC and NMDAR-eEPSC amplitudes ( \pm SEM) of CA1 pyramidal neurons expressing Tiam1 shRNA normalized to their respective control cell average eEPSC amplitudes. Tiam1 shRNA expression did not significantly affect AMPAR-eEPSC amplitude ( $n=9$ pairs) or NMDAR-eEPSC amplitude ( $n=8$ pairs) in CA1 pyramidal neurons. n.s., Not significant, Wilcoxon signed-rank test.

gest that Tiam1 plays a pathway-specific role in the regulation of glutamatergic synapse function in the hippocampus.

\section{Loss of Tiam 1 expression in DG granule neurons results in increased spine length}

RhoGEF proteins have been previously implicated in glutamatergic synapse regulation. Generally, these proteins influence synapse function through their ability to regulate actin polymerization in dendritic spines. The actin cytoskeleton in dendritic spines plays a critical role in determining spine morphology. Previous studies examining the function of synaptic regulatory RhoGEFs frequently report changes in dendritic spine number and morphology due to alterations of the synaptic actin cytoskeleton (Fu et al., 2007; Tolias et al., 2007; Herring and Nicoll, 2016). Tiam1 is a well known Rac1-specific RhoGEF (Habets et al., 1994; Michiels et al., 1995). Therefore, we reasoned that the reduction in AMPAR-eEPSC amplitude we observe following Tiam1 knockdown may stem from actin-mediated alterations of dendritic spine morphology. To quantify and compare the effect of Tiam1 knockdown on dendritic spine morphology in CA1 pyramidal and DG granule neurons, $z$-stacks of confocal images were collected, and spine-bearing dendrites were reconstructed (Fig. $3 A, B, D, E)$. We found that knocking down Tiam 1 expression in DG granule neurons had no effect on spine density ( $p=0.6$; for GFP, $n=19$ segments; for Tiam 1 shRNA, $n=17$ segments; Wilcoxon rank sum test; Fig. $3 B, C$ ). However, quantitative morphometric analysis revealed a significant increase in spine length, largely accounted for by an increase in spine neck length in DG granule neurons relative to GFP-expressing controls $(p=$ 0.00024 for spine length; $p=0.0014$ for spine neck length; for GFP, $n=19$ segments; for Tiam 1 shRNA, $n=17$ segments; Wilcoxon rank sum test; Fig. $3 B, C)$. Thus, Tiam1 knockdown in DG granule neurons causes spines to take on a filopodia-like appearance. Filopodia represent immature precursors of glutamatergic synapses exhibiting reduced AMPAR expression (Dailey and Smith, 1996; Durand et al., 1996; Matsuzaki et al., 2001; Okabe et al., 2001; Cohen-Cory, 2002). This finding is consistent with the reduction we observe in the number of synapses expressing AMPARs following Tiam1 knockdown (Fig. 1I,J). Together, our data suggest that the downregulation of Tiam1 function in DG granule neurons results in the inhibition of glutamatergic synapse function by impeding synaptic maturation. In marked contrast to DG granule neurons and consistent with our electrophysiological results, we found no significant differences in any spine parameters in Tiam 1 shRNA-expressing CA1 pyramidal neurons $(p=0.26$ for spine length; $p=0.55$ for spine neck length; $p=0.17$ for head volume; $p=0.7$ for spine density; for GFP, $n=12$ segments; for Tiam 1 shRNA, $n=25$ segments; Wilcoxon rank sum test; Fig. $3 E, F$ ).

To confirm that the effects we observe on glutamatergic synapses in DG granule neurons are due to the specific knockdown of Tiam1, we coexpressed Tiam1 shRNA with an shRNAresistant form of Tiam1 in DG granule neurons. We then performed paired recordings with DG granule neurons coexpressing 
A

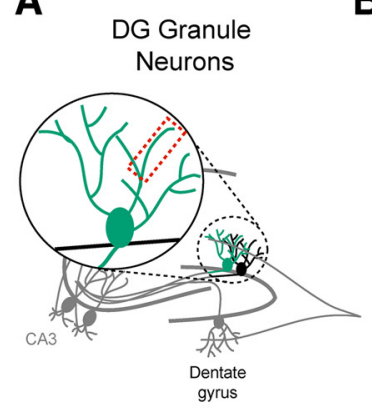

B

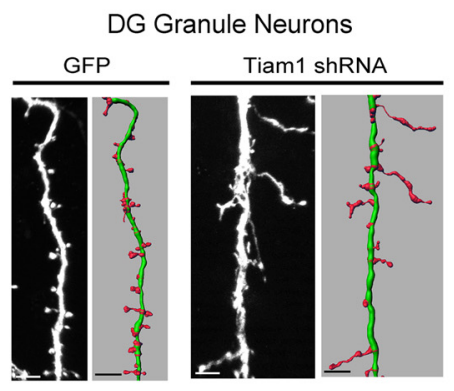

E

D

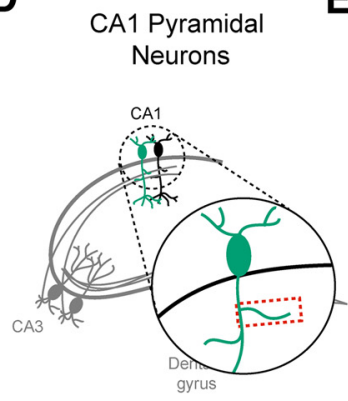

CA1 Pyramidal Neurons

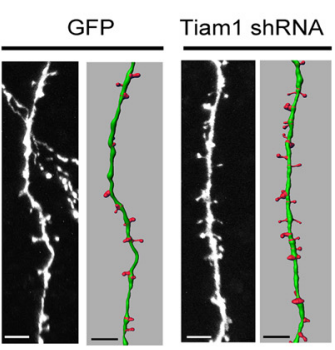

C

DG Granule Neurons
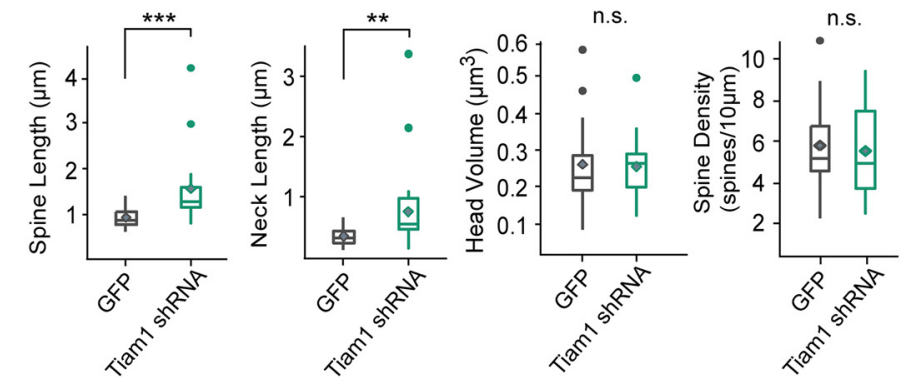

F

CA1 Pyramidal Neurons
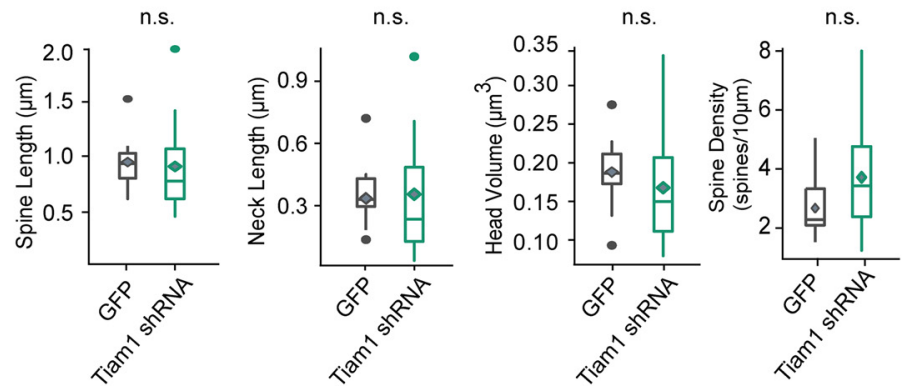

Figure 3. Tiam 1 knockdown increases dendritic spine length in DG granule neurons, but not in CA1 pyramidal neurons. $A, D$, Schematic representation of areas of image acquisition from DG granule neuron dendrites and apical CA1 pyramidal neuron dendrites, respectively. $\boldsymbol{B}, \boldsymbol{E}$, Representative dendritic segments and corresponding reconstructed segments from GFP and Tiam1 shRNA-expressing DG granule neurons and CA1 pyramidal neurons. Scale bars, $4 \mu \mathrm{m}$. $C, F$, Boxplots show significant differences in spine length and spine neck length in DG granule neurons expressing Tiam1 shRNA (colored boxes) compared with GFP-expressing control neurons (gray boxes; $p=0.00024$ for spine length; $p=0.0014$ for spine neck length; for GFP, $n=19$ segments; for Tiam 1 shRNA, $n=17$ segments), no significant differences in head volume or spine density were detected ( $p=0.9$ for head volume; $p=0.6$ for spine density; for GFP, $n=19$ segments; for Tiam1 shRNA, $n=17$ segments). No significant differences were detected in any spine parameters in Tiam1 shRNA-expressing spines in CA1 pyramidal neurons compared with corresponding GFP-expressing control neurons (for GFP, $n=12$ segments; for Tiam 1 shRNA, $n=25$ segments; $p=0.26$ for spine length; $p=0.55$ for spine neck length; $p=0.17$ for head volume; $p=0.7$ for spine density; Wilcoxon rank-sum test) ${ }^{* * *} p<0.001,{ }^{* *} p<0.01$, Wilcoxon rank-sum test. n.s., Not significant.

the Tiam1 shRNA and shRNA-resistant Tiam1. We found that AMPAR-eEPSC amplitude in these neurons was restored to wildtype levels $(n=10$ pairs; $p=0.56$, Wilcoxon signed-rank test; Fig. 4A,C). We then examined whether shRNA-resistant Tiam1 expression rescues the filopodia-like spine morphology observed with Tiam 1 knockdown. Analysis of dendritic segments from DG granule neurons coexpressing Tiam1 shRNA and shRNAresistant Tiam1 revealed no detectable differences in spine density or morphology compared with GFP-expressing controls ( $p=1$ for spine length; $p=0.96$ for spine neck length; $p=0.60$ for head volume; $p=0.14$ for spine density; for GFP, $n=28$ segments; for Tiam1 rescue, $n=29$ segments, Wilcoxon ranksum test; Fig. 4E,F). From these results, we conclude that the synaptic phenotypes observed in DG granule neurons following Tiam1 knockdown result from the targeted depletion of Tiam1.

Generally, RhoGEFs are believed to regulate synaptic function through their influence on the actin cytoskeleton. RhoGEF proteins promote actin polymerization by activating small GTPases through their GEF domains. However, a GEF-independent role for Tiam1 in neurons was recently reported (Tang et al., 2019). Does the ability of Tiam 1 to support glutamatergic synapse function in DG granule neurons depend on its ability to activate small GTPases? To answer this question, we generated a shRNAresistant Tiam1 construct lacking the DH domain (Tiam1 $\Delta \mathrm{DH}$ ). This domain alone binds directly to the small GTPase Racl and is required for the ability of Tiam1 to activate Rac1 and influence the actin cytoskeleton (Zheng et al., 1996; Worthylake et al., 2000; Karnoub et al., 2001). When expressed in heterologous cells, we find that our Tiam $1 \Delta \mathrm{DH}$ construct produces levels of protein expression that are comparable to our wild-type Tiam1 construct (Fig. $4 D$ ). We then examined whether Tiam1 $\Delta \mathrm{DH}$ rescues the Tiam1 knockdown phenotype. When Tiam1 shRNA and Tiam1 $\triangle \mathrm{DH}$ were coexpressed in DG granule neurons, we found that AMPAR-eEPSC amplitude was reduced to a degree very similar to that observed in DG granule neurons transfected with the Tiam1 shRNA alone ( $p=0.02148, n=13$, Wilcoxon signedrank test; Fig. $4 B, C$ ). Together, our data demonstrate that the region necessary for Tiam 1-mediated small GTPase activation is required for the influence of Tiam1 on glutamatergic synapse function in DG granule neurons.

\section{The $\mathrm{PH}_{\mathrm{n}}$-CC-Ex domain of Tiam1 negatively regulates Tiam1} function at DG granule synapses

It has been previously shown that increasing the expression levels of synaptic regulatory RhoGEFs (i.e., Kalirin-7 and Trio-9) in CA1 pyramidal neurons produces synaptic activity-dependent increases in the strength of glutamatergic neurotransmission (Herring and Nicoll, 2016; Sadybekov et al., 2017). Such effects have been used as evidence supporting the role of these RhoGEF proteins in bidirectional regulation of synaptic strength. In contrast to Kalirin-7 and Trio-9, the overexpression of Tiam 1 in CA1 pyramidal neurons was found to have no effect on AMPAR- 
A
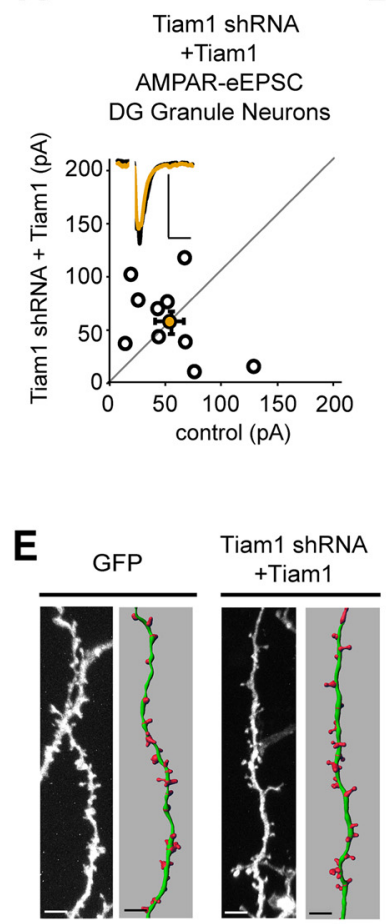

B

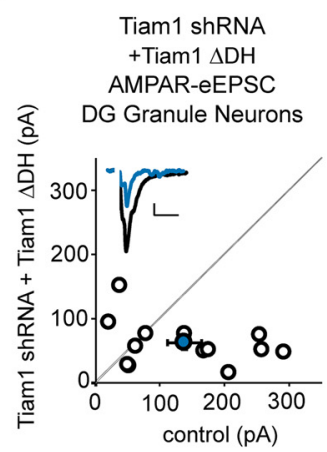

$\mathbf{F}$

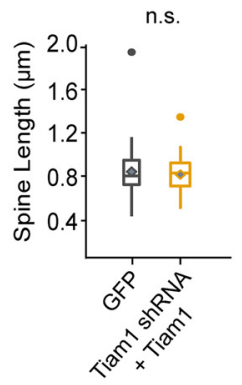

C

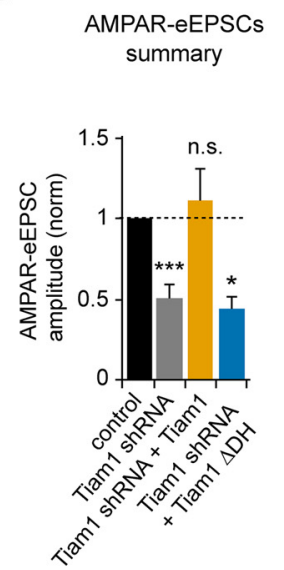

D

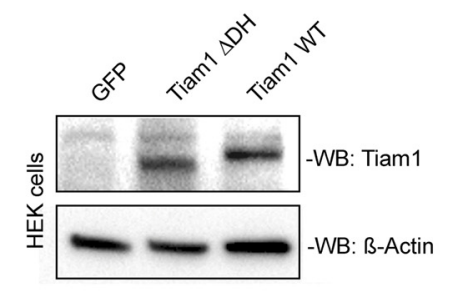

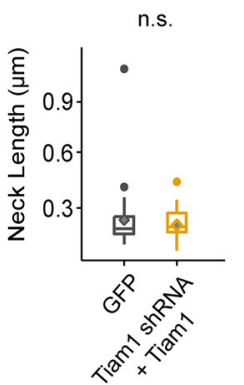
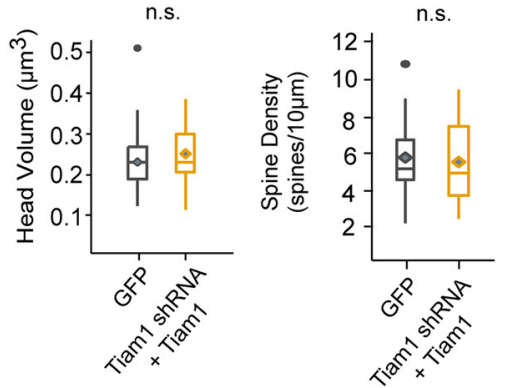

Figure 4. Full-length Tiam 1 but not Tiam $1 \triangle D$ H expression rescues Tiam 1 shRNA-mediated effects on glutamatergic synapses of DG granule neurons. $A, B$, Scatterplots with AMPAR-eEPSC and NMDAR-eEPSC amplitudes for DG granule neurons coexpressing Tiam1 shRNA and Tiam1 $\triangle \mathrm{DH}$, respectively, plotted against paired control neuron eEPSCs (open circles) with corresponding mean \pm SEM (filled circles). Barplots show average AMPAR-eEPSC and NMDAR-eEPSC amplitudes ( \pm SEM) of DG granule neurons expressing Tiam1 shRNA (gray bars) and DG granule neurons coexpressing Tiam1 shRNA, Tiam1 CDNA (yellow bars), and Tiam1 $\triangle \mathrm{DH}$ (blue bars) normalized to respective control cell average eEPSC amplitudes (black bar). ${ }^{* * *} p<0.001$, ${ }^{*} p<0.05$, Wilcoxon signed-rank test. $A$, Tiam 1 cDNA expression restores AMPAR-eEPSC amplitude in DG granule neurons coexpressing Tiam 1 shRNA ( $n=10$ pairs). Inset, Representative current traces from control and transfected (yellow) neurons stimulation artifacts removed. Calibration: 20 pA, 20 ms for AMPAR-eEPSC. B, Tiam1 $\Delta$ DH expression does not restore AMPAR-eEPSC $(p=0.02148, n=13$ pairs, Wilcoxon signed-rank test) and has no significant effect on NMDAR-eEPSC amplitudes in DG granule neurons coexpressing Tiam 1 shRNA ( $p=0.47, n=7$, Wilcoxon signed-rank test). Insets, Representative current traces from control and transfected (blue) neurons. Calibration: 20 pA, 20 ms for AMPAR-eEPSC. C, Average AMPAR-eEPSC and NMDAR-eEPSC amplitudes ( \pm SEM) of DG granule neurons expressing Tiam1 shRNA (gray bar) and DG granule neurons coexpressing Tiam1 shRNA, Tiam1 (yellow bar), and Tiam $1 \Delta$ DH normalized to respective control cell average eEPSC amplitudes (black bar). ${ }^{* *} p<0.001,{ }^{*} p<0.05$, Wilcoxon signed-rank test. $\boldsymbol{D}$, Western blot showing Tiam $1 \Delta \mathrm{DH}$ is expressed in HEK293 cells. $\boldsymbol{E}$, Representative dendritic segments and corresponding reconstructed filaments from control neurons expressing GFP and Tiam 1 and Tiam 1 shRNA-expressing DG granule neurons. Scale bars, $4 \mu \mathrm{m}$. $\boldsymbol{F}$, Boxplots show no detectable differences in any spine parameters in DG granule neurons coexpressing Tiam 1 and Tiam 1 shRNA (colored boxes) compared with GFP-expressing control neurons (gray boxes; $p=1$ for spine length; $p=0.96$ for spine neck length; $p=$ 0.60 for head volume; $p=0.14$ for spine density; for GFP, $n=28$ segments; for Tiam1 rescue, $n=29$ segments). n.s., Not significant, Wilcoxon rank-sum test.

eEPSC or NMDAR-eEPSC amplitude (Herring and Nicoll, 2016). Given that endogenous Tiaml plays a synaptic regulatory role in DG granule neurons, we were interested in whether increasing Tiam 1 expression and presumably function in DG granule neurons might lead to the strengthening of glutamatergic neurotransmission. To answer this question, we overexpressed wild-type Tiam1 (Fig. 5A) in DG granule neurons for $6 \mathrm{~d}$ before recording AMPAR-eEPSCs and NMDAR-eEPSCs in these neurons. We found that wild-type Tiam1 overexpression produced no change in AMPAR-eEPSC or NMDAR-eEPSC amplitudes (for AMPAR-eEPSCs: $p=0.22, n=14$ pairs; for NMDAR-eEPSCs: $p=0.38, n=7$ pairs; Wilcoxon signed-rank test; Fig. $5 B-E$ ). Unlike Kalirin and Trio, Tiam1 contains a $\mathrm{PH}_{\mathrm{n}}$-CC-Ex domain (Fig. $5 A$ ). It was recently shown that the GEF activity of Tiam 1 is auto-inhibited by this $\mathrm{PH}_{\mathrm{n}}$-CC-Ex domain (Matsuzawa et al., 2016; $\mathrm{Xu}$ et al., 2017). We wondered whether removing the $\mathrm{PH}_{\mathrm{n}}{ }^{-}$ CC-Ex domain from Tiam1 would allow Tiam1 overexpression to increase AMPAR-mediated neurotransmission at performant path-DG synapses. To answer this question, we generated a Tiam1 construct lacking the $\mathrm{PH}_{\mathrm{n}}-\mathrm{CC}-\mathrm{Ex}$ domain (Tiam1 $\triangle$ PHCCEx; Fig. $5 A$ ) and expressed this construct in dentate granule neurons of entorhino-hippocampal slices for $6 \mathrm{~d}$. In marked contrast to wild-type Tiam1, the overexpression of Tiam1 $\triangle$ PHCCEx in DG granule neurons produced a $70 \%$ increase in AMPAR-eEPSC amplitude ( $p=0.04, n=12$ pairs, Wilcoxon signed-rank test; Fig. $5 B, C$ ) No effect of Tiam $1 \triangle$ PHCCEx overexpression on NMDAR-eEPSC amplitude was observed ( $p=$ $0.50, n=8$ pairs, Wilcoxon signed-rank test; Fig. $5 D-E$ ). Such data suggest that the $\mathrm{PH}_{\mathrm{n}}$-CC-Ex domain of Tiam1 exerts a negative influence on Tiam1 function at DG granule neuron synapses and potentially reveal a mechanism by which synaptic Tiam 1 function is dynamically regulated.

\section{Discussion}

Previous studies have relied on dissociated hippocampal neurons to characterize the role of endogenous Tiam1 in glutamatergic synapse development (Chen and Macara, 2005; Tolias et al., 2005; Zhang and Macara, 2006; Um et al., 2014). In these preparations, glutamatergic synapses form between neurons of uncertain identity. Thus, the role of Tiam1 in synaptic maturation at naturally occurring glutamatergic synapses is unknown. In situ studies suggest Tiaml expression is enriched in granule neurons in the dentate gyrus (Lein et al., 2006), suggesting that Tiam1 regulation of glutamatergic neurotransmission may be most relevant at per- 
A
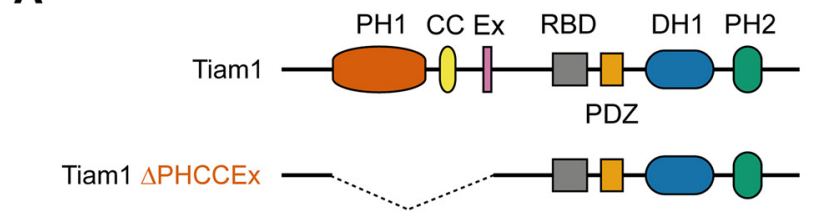

B

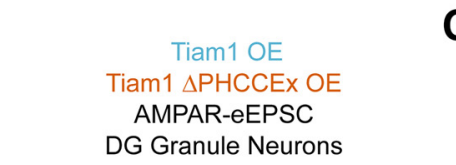
C
Tiam1 OE
Tiam1 $\triangle$ PHCCEX OE
Tiam1 $\triangle$ PHCCEX OE
AMPAR-eEPSC

DG Granule Neurons
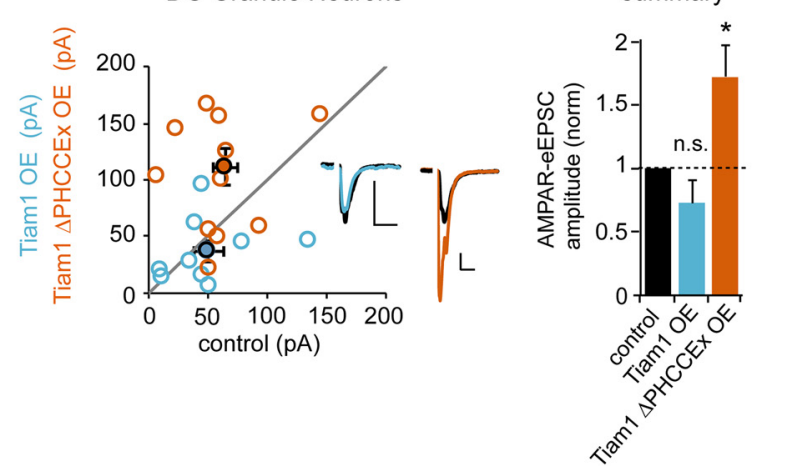

D
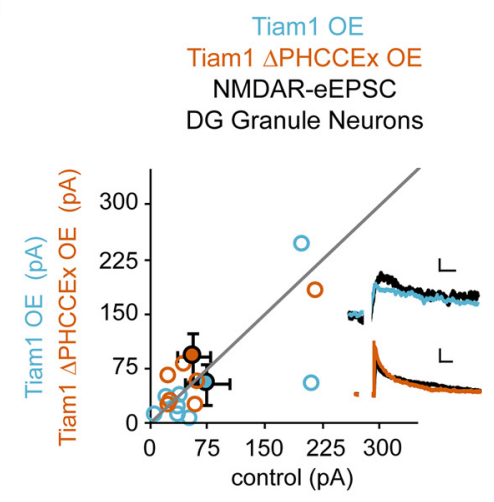

E

Tiam1 OE

Tiam1 $\triangle$ PHCCEX OE

NMDAR-eEPSC

summary

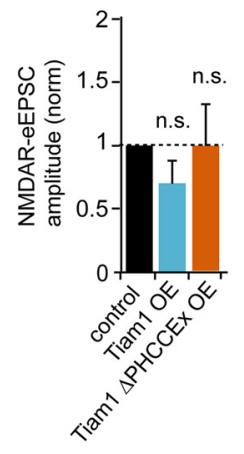

Figure 5. Tiam $1 \triangle P H C C$ Ex expression increases AMPAR-mediated neurotransmission in DG granule neurons. $A$, Illustration of the protein domains of Tiam1; full-length Tiam1 (top) and Tiam1 $\triangle \mathrm{PH}_{\mathrm{n}} \mathrm{CC}$-Ex (bottom). $\boldsymbol{B}, \boldsymbol{D}$, Scatterplots with AMPAR-eEPSC amplitudes for DG granule neurons expressing Tiam1 plotted against paired control neuron eEPSC (open circles) with corresponding mean \pm SEM (filled circles). Insets, Representative current traces from control and transfected (blue for Tiam1 $0 \mathrm{E}$; vermillion for Tiam $1 \triangle \mathrm{PH}_{\mathrm{n}}$-CC-Ex) neurons with stimulation artifacts removed. Calibration: $20 \mathrm{pA}$ for both AMPAR-eEPSC and NMDAR-eEPSC, $20 \mathrm{~ms}$ for AMPAR-eEPSC, $50 \mathrm{~ms}$ for NMDAR-eEPSC. $\boldsymbol{B}$, Tiam1 overexpression (OE) produces no detectable differences in AMPA-eEPSC amplitude ( $p=0.67, n=9$ pairs); Tiam1 $\triangle$ PHCCEx expression increases AMPAR-eEPSC amplitude ( $p=0.04, n=12$ pairs). $\boldsymbol{D}$, NMDAR-eEPSC amplitudes were not significantly affected in Tiam 1 or Tiam $1 \Delta \mathrm{PH}_{n}$-CC-Ex-expressing neurons and paired controls ( $p=0.38, n=7$ pairs for Tiam $10 \mathrm{E} ; p=0.50, n=8$ pairs for Tiam $1 \Delta$ PHCCEX). $\boldsymbol{C}, \boldsymbol{E}$, Bar plots of average AMPAR-eEPSC and NMDAR-eEPSC amplitudes ( \pm SEM) of DG granule neurons overexpressing Tiam 1 (blue for Tiam $10 \mathrm{O}$; vermillion for Tiam $1 \triangle \mathrm{PHCCEx}$ 0E) respective control cell average eEPSC amplitudes. Wilcoxon signed-rank test was used to compare across independent conditions. ${ }^{*} p<0.05$, n.s., Not significant, Wilcoxon signed-rank test.

forant path-DG synapses in the hippocampus. In the present study, we find that inhibition of Tiam1 function in DG granule neurons results in a significant reduction in glutamatergic synapse function. In marked contrast to dentate granule neurons, Tiam 1 expression appears to be very low in CA1 pyramidal neurons. Consistent with Tiam $1 \mathrm{mRNA}$ and protein expression data, we find that knocking down Tiam 1 in CA1 pyramidal neurons

has no impact on baseline glutamatergic neurotransmission at Schaffer collateral-CA1 synapses. Together, these results strongly support a pathway-specific role for Tiam1 in glutamatergic synapse regulation in the hippocampus. Going forward, it will be interesting to determine whether Tiam 1 differentially supports medial and lateral perforant pathway function in the dentate gyrus and whether glutamatergic pathways in other brain regions are regulated by Tiam1.

In addition to a reduction in synaptic AMPAR function, we found that knocking down Tiam1 expression in DG granule neurons resulted in the elongation of dendritic spines. This alteration of spine morphology was not observed following Tiam1 shRNA expression in CA1 pyramidal neurons. Tiam1 promotes Rac1 activation (Tolias et al., 2005). Racl activity is thought to be critical for the maturation of glutamatergic synapses, resulting in the conversion of immature elongated filopodia into shorter more mature spines (Nakayama and Luo, 2000; Tashiro and Yuste, 2004; De Rubeis et al., 2013). The elongated dendritic protrusions we observe following the reduction of Tiam1 function are consistent with descriptions of immature filopodial spines, suggesting that the inhibition of Tiam1 function results in the inhibition of glutamatergic synapse maturation. Filopodialike spines are proposed to be the structural correlates of NMDAR-expressing but AMPAR-lacking "silent" synapses that mature through the synaptic insertion of AMPARs (Dailey and Smith, 1996; Durand et al., 1996; Matsuzaki et al., 2001; Okabe et al., 2001; Cohen-Cory, 2002; Tian et al., 2018). Consistent with this idea, we performed coefficient of variation and failure analysis on AMPAR-eEPSCs from Tiam1 shRNA-expressing neurons and found that reductions in AMPAR-eEPSC amplitude in these neurons result from a decrease in the number of synapses expressing AMPA receptors. The inhibition of Tiam 1 function in DG granule neurons did not affect presynaptic neurotransmitter release or NMDAR-eEPSC amplitude. Together, these data strongly suggest that the inhibition of Tiam1 function increases the number of silent synapses of DG granule neurons and are consistent with our morphological data supporting a role for Tiam 1 in perforant path-DG synapse maturation. It will now be important to identify which Tiam1-dependent synaptic regulatory pathways are required for normal glutamatergic synapse maturation in DG granule neurons. It will be particularly interesting to examine the role of Tiam 1 in glutamatergic synapse plasticity. While the present study was under review, a study was published where Tiam1 knockdown was shown to inhibit structural long-term potentiation (sLTP) in CA1 pyramidal neurons (Saneyoshi et al., 2019). Surprisingly, this finding suggests that Tiam1 plays an important role in activity-dependent structural changes of synapses in CA1 pyramidal neurons despite very low expression in these cells. The outcome of future studies comparing the role of Tiam1 in functional LTP in dentate granule and CA1 pyramidal neurons will be of great interest.

Generally, the influence of Tiam1 is thought to be through GEF domain-mediated regulation of the actin cytoskeleton. The GEF domain of Tiam 1 activates the small GTPase Rac1, which ultimately promotes actin polymerization. However, it was recently reported that Tiam 1 is able to influence neuronal morphology through a GEF-independent mechanism (Tang et al., 2019). To determine whether the GEF domain is required for the influence of Tiam 1 on glutamatergic synapse function in the dentate gyrus, we engineered a mutant form of Tiam1 lacking its GEF/DH domain (Tiam1 $\Delta \mathrm{DH}$ ). This mutation eliminates the entire binding site for Racl (Worthylake et al., 2000) and thus represents a truly "GEF-dead" form of Tiam1. Despite exhibiting 
a level of expression comparable to wild-type Tiam1, we found that Tiam $1 \Delta \mathrm{DH}$ cannot rescue the synaptic phenotype produced by knocking down Tiam 1 expression. Thus, the support by Tiam1 of synaptic function in dentate granule neurons is dependent on an intact GEF domain. The most cogent explanation for this observation is that Tiaml's support of perforant path-DG synapse function is dependent on the ability of Tiam 1 to activate small GTPases like Rac1. However, we acknowledge that we cannot rule out alternative explanations such as protein misfolding or improper trafficking that might be produced by deleting the GEF/DH domain.

Previously, the overexpression of synaptic regulatory RhoGEF proteins in CA1 pyramidal neurons was shown to result in a significant increase in synaptic AMPAR function (Herring and Nicoll, 2016). The overexpression of Tiam1 in CA1 pyramidal neurons, on the other hand, was found to have no impact on Schaffer collateral-CA1 synapse function. Here, we overexpressed Tiam1 in DG granule neurons to determine whether elevated Tiam1 function can augment the strength of perforant path-DG neurotransmission. We found that the function of these synapses was also unaffected by Tiam 1 overexpression. One explanation as to why Tiam1 overexpression fails to increase glutamatergic neurotransmission in DG granule neurons is that Tiam1 may exist in an inactive state in neurons and require additional molecular mechanisms for activation (Fleming et al., 1999; Crompton et al., 2000; Mertens et al., 2003; Terawaki et al., 2010). In this case, a rate-limiting molecular mechanism may exist within neurons preventing excess Tiam 1 function at synapses following overexpression. Previous in vitro studies have shown that the $\mathrm{PH}_{\mathrm{n}}$-CC-Ex domain of Tiam1 can negatively regulate Tiam1 GEF activity, suggesting that this domain is autoinhibitory (Xu et al., 2017). Consistent with this idea, we found that the expression of a Tiam1 mutant lacking the conserved $\mathrm{PH}_{\mathrm{n}}$-CC-Ex domain produced an increase in AMPAR-eEPSC amplitude. Our data suggest a potential role of the $\mathrm{PH}_{\mathrm{n}}-\mathrm{CC}$-Ex in auto-inhibition of Tiam 1 and reveals a possible regulatory mechanism that permits Tiam1-mediated increases in glutamatergic synapse strength.

Recent evidence suggests that glutamatergic synapses exhibit remarkable molecular diversity in the brain, with commonly studied Schaffer collateral-CA1 synapses differing substantially from perforant path-DG synapses of the hippocampus. However, we know very little about the unique regulatory mechanisms that exist at perforant path-DG synapses. The present study demonstrates that perforant path-DG synapses are under the control of a RhoGEF-mediated molecular program that is distinct from Schaffer collateral-CA1 glutamatergic synapses in the hippocampus. Going forward, it will be interesting to determine whether various RhoGEF proteins are a part of a unique complement of synaptic proteins that are specific to different glutamatergic synapse subtypes. Moreover, differential expression of RhoGEF proteins may define distinct molecular and functional glutamatergic synapse subtypes in the brain. RhoGEF proteins seem particularly well suited for this task. The RhoGEF protein family has many members that exhibit considerable variability outside of their GTPase activation (GEF) domains, suggesting unique molecular regulatory mechanisms are likely to govern the function of specific RhoGEF proteins (Schmidt and Hall, 2002). In future studies, it may be useful to look to specific RhoGEF proteins as potential targets for treating brain-related disorders that stem from glutamatergic synapse dysfunction in specific regions of the brain.

\section{References}

Bekkers JM, Stevens CF (1990) Presynaptic mechanism for long-term potentiation in the hippocampus. Nature 346:724-729.

Bonnici B, Kapfhammer JP (2009) Modulators of signal transduction pathways can promote axonal regeneration in entorhino-hippocampal slice cultures. Eur J Pharmacol 612:35-40.

Cembrowski MS, Wang L, Sugino K, Shields BC, Spruston N (2016) Hipposeq: a comprehensive RNA-seq database of gene expression in hippocampal principal neurons. eLife 5:e14997.

Chen X, Macara IG (2005) Par-3 controls tight junction assembly through the rac exchange factor Tiaml. Nat Cell Biol 7:262-269.

Cohen-Cory S (2002) The developing synapse: construction and modulation of synaptic structures and circuits. Science 298:770-776.

Coultrap SJ, Nixon KM, Alvestad RM, Valenzuela CF, Browning MD (2005) Differential expression of NMDA receptor subunits and splice variants among the CA1, CA3 and dentate gyrus of the adult rat. Brain Res Mol Brain Res 135:104-111.

Crompton AM, Foley LH, Wood A, Roscoe W, Stokoe D, McCormick F, Symons M, Bollag G (2000) Regulation of Tiam1 nucleotide exchange activity by pleckstrin domain binding ligands. J Biol Chem 275:25751-25759.

Dailey ME, Smith SJ (1996) The dynamics of dendritic structure in developing hippocampal slices. J Neurosci 16:2983-2994.

Datson NA, Meijer L, Steenbergen PJ, Morsink MC, Van Der Laan S, Meijer OC, de Kloet ER (2004) Expression profiling in laser-microdissected hippocampal subregions in rat brain reveals large subregion-specific differences in expression. Eur J Neurosci 20:2541-2554.

Datson NA, Morsink MC, Steenbergen PJ, Aubert Y, Schlumbohm C, Fuchs E, de Kloet ER (2009) A molecular blueprint of gene expression in hippocampal subregions CA1, CA3, and DG is conserved in the brain of the common marmoset. Hippocampus 19:739-752.

del Castillo J, Katz B (1954) Quantal components of the end-plate potential. J Physiol 124:560-573.

De Rubeis S, Pasciuto E, Li KW, Fernández E, Di Marino D, Buzzi A, Ostroff LE, Klann E, Zwartkruis FJ, Komiyama NH, Grant SG, Poujol C, Choquet D, Achsel T, Posthuma D, Smit AB, Bagni C (2013) CYFIP1 coordinates mRNA translation and cytoskeleton remodeling to ensure proper dendritic spine formation. Neuron 79:1169-1182.

Duman JG, Tzeng CP, Tu YK, Munjal T, Schwechter B, Ho TS, Tolias KF (2013) The adhesion-GPCR BAI1 regulates synaptogenesis by controlling the recruitment of the Par3/Tiam1 polarity complex to synaptic sites. J Neurosci 33:6964-6978.

Durand GM, Kovalchuk Y, Konnerth A (1996) Long-term potentiation and functional synapse induction in developing hippocampus. Nature 381: 71-75.

Ehler E, van Leeuwen F, Collard JG, Salinas PC (1997) Expression of tiam-1 in the developing brain suggests a role for the tiam-1-rac signaling pathway in cell migration and neurite outgrowth. Mol Cell Neurosci 9:1-12.

Elias GM, Elias LA, Apostolides PF, Kriegstein AR, Nicoll RA (2008) Differential trafficking of AMPA and NMDA receptors by SAP102 and PSD-95 underlies synapse development. Proc Natl Acad Sci U S A 105:2095320958.

Fleming IN, Elliott CM, Buchanan FG, Downes CP, Exton JH (1999) Ca2+/ calmodulin-dependent protein kinase II regulates Tiam1 by reversible protein phosphorylation. J Biol Chem 274:12753-12758.

Fu WY, Chen Y, Sahin M, Zhao XS, Shi L, Bikoff JB, Lai KO, Yung WH, Fu AK, Greenberg ME, Ip NY (2007) Cdk5 regulates EphA4-mediated dendritic spine retraction through an ephexin1-dependent mechanism. Nat Neurosci 10:67-76.

Goold CP, Nicoll RA (2010) Single-cell optogenetic excitation drives homeostatic synaptic depression. Neuron 68:512-528.

Gray JA, Shi Y, Usui H, During MJ, Sakimura K, Nicoll RA (2011) Distinct modes of AMPA receptor suppression at developing synapses by GluN2A and GluN2B: single-cell NMDA receptor subunit deletion in vivo. Neuron 71:1085-1101.

Habets GG, Scholtes EH, Zuydgeest D, van der Kammen RA, Stam JC, Berns A, Collard JG (1994) Identification of an invasion-inducing gene, Tiam-1, that encodes a protein with homology to GDP-GTP exchangers for Rho-like proteins. Cell 77:537-549.

Hamilton AM, Lambert JT, Parajuli LK, Vivas O, Park DK, Stein IS, Jahncke JN, Greenberg ME, Margolis SS, Zito K (2017) A dual role for the Rho- 
GEF Ephexin5 in regulation of dendritic spine outgrowth. Mol Cell Neurosci 80:66-74.

Herring BE, Nicoll RA (2016) Kalirin and trio proteins serve critical roles in excitatory synaptic transmission and LTP. Proc Natl Acad Sci U S A 113:2264-2269.

Incontro S, Díaz-Alonso J, Iafrati J, Vieira M, Asensio CS, Sohal VS, Roche KW, Bender KJ, Nicoll RA (2018) The CaMKII/NMDA receptor complex controls hippocampal synaptic transmission by kinase-dependent and independent mechanisms. Nat Commun 9:2069.

Kang H, Schuman EM (1995) Long-lasting neurotrophin-induced enhancement of synaptic transmission in the adult hippocampus. Science 267:1658-1662.

Karnoub AE, Worthylake DK, Rossman KL, Pruitt WM, Campbell SL, Sondek J, Der CJ (2001) Molecular basis for Racl recognition by guanine nucleotide exchange factors. Nat Struct Biol 8:1037-1041.

Ke MT, Fujimoto S, Imai T (2013) SeeDB: a simple and morphologypreserving optical clearing agent for neuronal circuit reconstruction. Nature Neuroscience 16:1154-1161.

Kokaia M, Asztely F, Olofsdotter K, Sindreu CB, Kullmann DM, Lindvall O (1998) Endogenous neurotrophin-3 regulates short-term plasticity at lateral perforant path-granule cell synapses. J Neurosci 18:8730-8739.

Lein ES, Zhao X, Gage FH (2004) Defining a molecular atlas of the hippocampus using DNA microarrays and high-throughput in situ hybridization. J Neurosci 24:3879-3889.

Lein ES, Hawrylycz MJ, Ao N, Ayres M, Bensinger A, Bernard A, Boe AF, Boguski MS, Brockway KS, Byrnes EJ, Chen L, Chen L, Chen TM, Chin MC, Chong J, Crook BE, Czaplinska A, Dang CN, Datta S, Dee NR, et al (2006) Genome-wide atlas of gene expression in the adult mouse brain. Nature 445:168.

Levy JM, Chen X, Reese TS, Nicoll RA (2015) Synaptic consolidation normalizes AMPAR quantal size following MAGUK loss. Neuron 87:534548.

Lu W, Shi Y, Jackson AC, Bjorgan K, During MJ, Sprengel R, Seeburg PH, Nicoll RA (2009) Subunit composition of synaptic AMPA receptors revealed by a single-cell genetic approach. Neuron 62:254-268.

Malinow R, Tsien RW (1990) Presynaptic enhancement shown by wholecell recordings of long-term potentiation in hippocampal slices. Nature 346:177-180.

Margolis SS, Salogiannis J, Lipton DM, Mandel-Brehm C, Wills ZP, Mardinly AR, Hu L, Greer PL, Bikoff JB, Ho HY, Soskis MJ, Sahin M, Greenberg ME (2010) EphB-mediated degradation of the RhoA GEF Ephexin5 relieves a developmental brake on excitatory synapse formation. Cell 143:442-455.

Martin-Vilchez S, Whitmore L, Asmussen H, Zareno J, Horwitz R, NewellLitwa K (2017) RhoGTPase regulators orchestrate distinct stages of synaptic development. PLoS One 12:e0170464.

Matsuzaki M, Ellis-Davies GC, Nemoto T, Miyashita Y, Iino M, Kasai H (2001) Dendritic spine geometry is critical for AMPA receptor expression in hippocampal CA1 pyramidal neurons. Nat Neurosci 4:10861092.

Matsuzawa K, Akita H, Watanabe T, Kakeno M, Matsui T, Wang S, Kaibuchi K (2016) PAR3-aPKC regulates Tiam 1 by modulating suppressive internal interactions. Mol Biol Cell 27:1511-1523.

Mertens AE, Roovers RC, Collard JG (2003) Regulation of Tiam1-rac signalling. FEBS Lett 546:11-16.

Michiels F, Habets GG, Stam JC, van der Kammen RA, Collard JG (1995) A role for rac in Tiam1-induced membrane ruffling and invasion. Nature 375:338-340.

Miller MB, Yan Y, Eipper BA, Mains RE (2013) Neuronal rho GEFs in synaptic physiology and behavior. Neuroscientist 19:255-273.

Nakayama AY, Luo L (2000) Intracellular signaling pathways that regulate dendritic spine morphogenesis. Hippocampus 10:582-586.

Okabe S, Miwa A, Okado H (2001) Spine formation and correlated assembly of presynaptic and postsynaptic molecules. J Neurosci 21:6105-6114.

Penzes P, Rafalovich I (2012) Regulation of the actin cytoskeleton in dendritic spines. Adv Exp Med Biol 970:81-95.

Penzes P, Beeser A, Chernoff J, Schiller MR, Eipper BA, Mains RE, Huganir RL (2003) Rapid induction of dendritic spine morphogenesis by transsynaptic ephrinB-EphB receptor activation of the rho-GEF kalirin. Neuron 37:263-274.

Penzes P, Cahill ME, Jones KA, Srivastava DP (2008) Convergent CaMK and RacGEF signals control dendritic structure and function. Trends Cell Biol 18:405-413.

Prang P, Del Turco D, Kapfhammer JP (2001) Regeneration of entorhinal fibers in mouse slice cultures is age dependent and can be stimulated by NT-4, GDNF, and modulators of G-proteins and protein kinase C. Exp Neurol 169:135-147.

Ryan TJ, Roy DS, Pignatelli M, Arons A, Tonegawa S (2015) Memory. engram cells retain memory under retrograde amnesia. Science 348 : 1007-1013.

Sadybekov A, Tian C, Arnesano C, Katritch V, Herring BE (2017) An autism spectrum disorder-related de novo mutation hotspot discovered in the GEF1 domain of Trio. Nat Commun 8:601.

Saneyoshi T, Matsuno H, Suzuki A, Murakoshi H, Hedrick NG, Agnello E, O'Connell R, Stratton MM, Yasuda R, Hayashi Y (2019) Reciprocal activation within a kinase-effector complex underlying persistence of structural LTP. Neuron 102:1199-1210.e6.

Schmidt A, Hall A (2002) Guanine nucleotide exchange factors for rho GTPases: turning on the switch. Genes Dev 16:1587-1609.

Schnell E, Sizemore M, Karimzadegan S, Chen L, Bredt DS, Nicoll RA (2002) Direct interactions between PSD-95 and stargazin control synaptic AMPA receptor number. Proc Natl Acad Sci U S A 99:13902-13907.

Stoppini L, Buchs PA, Muller D (1991) A simple method for organotypic cultures of nervous tissue. J Neurosci Methods 37:173-182.

Tang LT, Diaz-Balzac CA, Rahman M, Ramirez-Suarez NJ, Salzberg Y, Lázaro-Peña MI, Bülow HE (2019) TIAM-1/GEF can shape somatosensory dendrites independently of its GEF activity by regulating F-actin localization. Elife 8:e38949.

Tashiro A, Yuste R (2004) Regulation of dendritic spine motility and stability by Racl and rho kinase: evidence for two forms of spine motility. Mol Cell Neurosci 26:429-440.

Terawaki S, Kitano K, Mori T, Zhai Y, Higuchi Y, Itoh N, Watanabe T, Kaibuchi K, Hakoshima T (2010) The PHCCEx domain of Tiam $1 / 2$ is a novel protein- and membrane-binding module. EMBO J 29:236-250.

Tian C, Kay Y, Sadybekov A, Rao S, Katritch V, Herring BE (2018) An Intellectual Disability-Related Missense Mutation in Racl Prevents LTP Induction. Front Mol Neurosci 11:223.

Tolias KF, Bikoff JB, Burette A, Paradis S, Harrar D, Tavazoie S, Weinberg RJ, Greenberg ME (2005) The Rac1-GEF Tiam1 couples the NMDA receptor to the activity-dependent development of dendritic arbors and spines. Neuron 45:525-538.

Tolias KF, Bikoff JB, Kane CG, Tolias CS, Hu L, Greenberg ME (2007) The Rac1 guanine nucleotide exchange factor Tiam 1 mediates EphB receptordependent dendritic spine development. Proc Natl Acad Sci US A 104:7265-7270.

Um K, Niu S, Duman JG, Cheng JX, Tu YK, Schwechter B, Liu F, Hiles L, Narayanan AS, Ash RT, Mulherkar S, Alpadi K, Smirnakis SM, Tolias KF (2014) Dynamic control of excitatory synapse development by a Rac1 GEF/GAP regulatory complex. Dev Cell 29:701-715.

Worthylake DK, Rossman KL, Sondek J (2000) Crystal structure of Racl in complex with the guanine nucleotide exchange region of Tiam1. Nature 408:682-688.

Xie Z, Srivastava DP, Photowala H, Kai L, Cahill ME, Woolfrey KM, Shum CY, Surmeier DJ, Penzes P (2007) Kalirin-7 controls activity-dependent structural and functional plasticity of dendritic spines. Neuron 56:640 656.

Xu Z, Gakhar L, Bain FE, Spies M, Fuentes EJ (2017) The Tiam1 guanine nucleotide exchange factor is auto-inhibited by its pleckstrin homology coiled-coil extension domain. J Biol Chem 292:17777-17793.

Zhang H, Macara IG (2006) The polarity protein PAR-3 and TIAM1 cooperate in dendritic spine morphogenesis. Nat Cell Biol 8:227-237.

Zhao X, Lein ES, He A, Smith SC, Aston C, Gage FH (2001) Transcriptional profiling reveals strict boundaries between hippocampal subregions. J Comp Neurol 441:187-196.

Zheng Y, Zangrilli D, Cerione RA, Eva A (1996) The pleckstrin homology domain mediates transformation by oncogenic dbl through specific intracellular targeting. J Biol Chem 271:19017-19020.

Zhu F, Cizeron M, Qiu Z, Benavides-Piccione R, Kopanitsa MV, Skene NG, Koniaris B, DeFelipe J, Fransén E, Komiyama NH, Grant SGN (2018) Architecture of the mouse brain synaptome. Neuron 99:781-799.e10. 